\title{
An Analytical and Approximate Solution for Nonlinear Volterra Partial Integro-Differential Equations with a Weakly Singular Kernel Using the Fractional Differential Transform Method
}

\author{
Rezvan Ghoochani-Shirvan, ${ }^{1}$ Jafar Saberi-Nadjafi ${ }^{D},{ }^{2}$ and Morteza Gachpazan ${ }^{2}$ \\ ${ }^{1}$ Department of Applied Mathematics, Ferdowsi University of Mashhad, International Campus, Mashhad, Iran \\ ${ }^{2}$ Department of Applied Mathematics, School of Mathematical Sciences, Ferdowsi University of Mashhad, Mashhad, Iran \\ Correspondence should be addressed to Jafar Saberi-Nadjafi; najafil41@gmail.com
}

Received 17 August 2017; Revised 24 November 2017; Accepted 18 January 2018; Published 1 April 2018

Academic Editor: Jaume Giné

Copyright (c) 2018 Rezvan Ghoochani-Shirvan et al. This is an open access article distributed under the Creative Commons Attribution License, which permits unrestricted use, distribution, and reproduction in any medium, provided the original work is properly cited.

\begin{abstract}
An analytical-approximate method is proposed for a type of nonlinear Volterra partial integro-differential equations with a weakly singular kernel. This method is based on the fractional differential transform method (FDTM). The approximate solutions of these equations are calculated in the form of a finite series with easily computable terms. The analytic solution is represented by an infinite series. We state and prove a theorem regarding an integral equation with a weak kernel by using the fractional differential transform method. The result of the theorem will be used to solve a weakly singular Volterra integral equation later on.
\end{abstract}

\section{Introduction}

Many engineering and physical problems result in the analysis of the nonlinear weakly singular Volterra integral equations (WSVIEs). These equations are applied in many areas [1] such as reaction-diffusion problems in small cells [2], theory of elasticity, heat conductions, hydrodynamics, stereology [3], the radiation of heat from semi-infinite solids [4], and other applications. Such equations have been studied by several authors [5-14].

The aim of this paper is applying the fractional differential transform method (FDTM) for solving WSVIE. The fractional differential transform method has recently been developed for solving the differential and integral equations. For example, in [15], FDTM is applied for fractional differential equations and in [16] it is used for fractional integrodifferential equations. This method is applied to nonlinear fractional partial differential equations in [17]. The use of the differential transform method (DTM) in electric circuit analysis was first proposed by Zhou [18].

The main challenge of partial integro-differential equations (PIDEs) with a weakly singular kernel is faced when we are looking for an analytical solution. By applying the differential transform method, the result mostly obtained is an analytical solution in the form of a polynomial. The differential transform method is different from the traditional high order Taylor series method, which requires symbolic competition of the necessary derivatives of the data functions. Making use of this method enables us to obtain highly accurate results or exact solutions for a partial integro-differential equation. The use of application of DTM and FDTM does not require linearization, discretization, or perturbation in contrast to the methods discussed in the literature $[8,9,19]$.

The form of WSVIE that we will consider in this paper with FDTM is

$$
\begin{aligned}
\phi_{t}= & \phi(x, t)+f(x, t) \\
& +\int_{0}^{t} \int_{0}^{x}(x-\xi)^{p-1} N[\phi(\xi, \eta)] d \xi d \eta,
\end{aligned}
$$

where $0<p<1,0 \leq \xi \leq x, 0 \leq \eta \leq t$, and $(x, t) \in[0,1] \times$ $[0,1]$ with the initial condition

$$
\phi(x, 0)=\phi_{0}(x),
$$


where $\phi$ is an unknown function in $\Lambda(=[0,1] \times[0,1])$ which should be determined and $f(x, t), \phi_{0}(x)$ are known functions and $N$ is a nonlinear operator. The given functions $f(x, t)$ and $\phi(x, t)$ are assumed to be sufficiently smooth in order to guarantee the existence and uniqueness of a solution $\phi \epsilon$ $C(\Lambda)$. It is assumed that the nonlinear term $N[\phi]$ satisfies the Lipschitz condition in $L^{2}(\Lambda)$.

The numerical treatment of (1) is not simple because the solutions of WSVIEs usually have a weak singularity at $x=0$. Different numerical techniques have been developed for the solution of PIDEs [8, 10, 20-25]. In this article, FDTM is applied to solve (1) and the main theorem is proved on the two-dimensional FDTM, while the one-dimensional FDTM has been applied in [26].

The paper is organized as follows: In Section 2, Caputo and Riemann-Liouville fractional derivatives are introduced. In Section 3, the theorems of the fractional differential transform method, preliminaries, and notations are explained. In Section 4, we have proposed the main theorem, for which a WSVIE can be considered as a series of FDT. Further, some examples of the application of FDTM are demonstrated, which show the accuracy of the method, in Section 5. We conclude our discussion in Section 6.

\section{Riemann-Liouville and Caputo Fractional Derivatives}

There are different kinds of definitions for the fractional derivative of order $q>0$; among various definitions of fractional derivatives of order $q>0$, the RiemannLiouville and Caputo formulas are the most common [27]. The Riemann-Liouville fractional integration of order $q$ is defined as

$$
J_{x_{0}}^{q} f(x)=\frac{1}{\Gamma(q)} \int_{x_{0}}^{x}(x-t)^{q-1} f(t) d t
$$

$$
q>0, x>x_{0}
$$

The following equations define Riemann-Liouville and Caputo fractional derivatives of order $q$, respectively:

$$
\begin{aligned}
D_{x_{0}}^{q} f(x) & =\frac{d^{m}}{d x^{m}}\left[J_{x_{0}}^{m-q} f(x)\right], \\
D_{* x_{0}}^{q} f(x) & =J_{x_{0}}^{m-q}\left[\frac{d^{m}}{d x^{m}} f(x)\right],
\end{aligned}
$$

where $m-1 \leq q<m$ and $m \in N$. From (4) and (5), we have

$$
D_{x_{0}}^{q} f(x)=\frac{1}{\Gamma(m-q)} \frac{d^{m}}{d x^{m}} \int_{x_{0}}^{x}(x-t)^{m-q-1} f(t) d t,
$$

$$
x>x_{0}
$$

\section{The Fractional Differential Transform Method (FDTM)}

There are some approaches to the generalization of the notion of differentiation to fractional orders. According to the
Riemann-Liouville formula, the fractional differentiation is defined by (6). The analytical and continuous function $f(x)$ is expended in terms of a fractional power series as follows:

$$
f(x)=\sum_{k=0}^{\infty} F(k)\left(x-x_{0}\right)^{k / \alpha}
$$

where $\alpha$ is the order of fraction and $F(k)$ is the fractional differential transform of $f(x)[15,28-30]$. In Caputo sense $[31],(6)$ is modified to handle integer-order initial conditions as follows:

$$
\begin{aligned}
D^{q} x_{0}\left[f(x)-\sum_{k=0}^{m-1} \frac{1}{k !}\left(x-x_{0}\right)^{k} f^{(k)}\left(x_{0}\right)\right]=\frac{1}{\Gamma(m-q)} \\
\cdot \frac{d^{m}}{d x^{m}}\left\{\int_{0}^{x}\left[\frac{f(t)-\sum_{k=0}^{m-1}(1 / k !)\left(t-x_{0}\right)^{k} f^{(k)}\left(x_{0}\right)}{(x-t)^{1+q-m}}\right] d t\right\} .
\end{aligned}
$$

Since the initial conditions are implemented to the integerorder derivatives, the transformation of the initial conditions also can be represented as follows:

$$
F(k)= \begin{cases}\frac{1}{(k / \alpha) !}\left[\frac{d^{k / \alpha} f(x)}{d x^{k / \alpha}}\right]_{x=x_{0}} & \text { if } \frac{k}{\alpha} \in N, \\ 0 & \text { if } \frac{k}{\alpha} \notin N,\end{cases}
$$

for $k=0,1,2, \ldots,(n \alpha-1)$, where $n$ is the order of FDE that is considered.

Consider a function $u(x, t)$ of two variables, and assume that it can be expressed as a product of two single-variable functions as $u(x, t)=f(x) g(t)$. The expansion of the function $u(x, t)$ in a Taylor series around a point $\left(x_{0}, t_{0}\right)$ is as follows:

$$
u(x, t)=\sum_{k=0}^{\infty} \sum_{h=0}^{\infty} U(k, h)\left(x-x_{0}\right)^{k / \beta}\left(t-t_{0}\right)^{h / \alpha} .
$$

If we take $\left(x_{0}, t_{0}\right)$ as $(0,0)$, then (10) can be illustrated as

$$
u(x, t)=\sum_{k=0}^{\infty} \sum_{h=0}^{\infty} U(k, h)(x)^{k / \beta}(t)^{h / \alpha},
$$

where $\alpha, \beta$ are the order of the fractions, $\alpha, \beta \in N$, and $U(k, h)=F(k) G(h)$ is called the spectrum of $u(x, t)$ and defined by

$$
\begin{aligned}
& U(k, h) \\
& \quad=\frac{1}{\Gamma(k / \beta+1) \Gamma(h / \alpha+1)}\left[D^{k / \beta} D^{h / \alpha} u(x, t)\right]_{\left(x_{0}, t_{0}\right)} .
\end{aligned}
$$

If we choose $\alpha=1$ and $\beta=1$, the fractional twodimensional differential transform reduces to the classical two-dimensional differential transform. Using (11) and (12), the theorems of FDTM are introduced as follows. The proofs of these theorems can be found in $[15,17]$.

Theorem 1. Suppose that $W(k, h), U(k, h)$, and $V(k, h)$ are the differential transformations of the functions $w(x, t), u(x, t)$, and $v(x, t)$, respectively, with order of fraction $\alpha$ and $\beta$; then 
(1) if $w(x, t)=u(x, t) \pm v(x, t)$, then $W(k, h)=U(k, h) \pm$ $V(k, h)$;

(2) if $w(x, t)=\lambda u(x, t)$, then $W(k, h)=\lambda U(k, h)$;

(3) if $w(x, t)=\left(x-x_{0}\right)^{p}\left(t-t_{0}\right)^{q}$, then

$$
\begin{aligned}
W(k, h) & =\delta(k-\beta p) \delta(h-\alpha q) \\
& = \begin{cases}1 & k=\beta p, h=\alpha q \\
0 & \text { otherwise }\end{cases}
\end{aligned}
$$

(4) if $w(x, t)=u(x, t) v(x, t)$, then

$$
\begin{aligned}
W(k, h) & =U(k, h) V(k, h) \\
& =\sum_{r=0}^{k} \sum_{s=0}^{h} U(r, h-s) V(k-r, s) ;
\end{aligned}
$$

(5) if $w(x, t)=x^{p} \sin (a t+b)$, then

$$
W(k, h)=\frac{a^{h}}{h !} \delta(k-p) \sin \left(\frac{h \pi}{2}+b\right) ;
$$

(6) if $w(x, t)=x^{p} \cos (a t+b)$, then

$$
W(k, h)=\frac{a^{h}}{h !} \delta(k-p) \cos \left(\frac{h \pi}{2}+b\right) ;
$$

(7) if $w(x, t)=x^{p} \exp (\lambda t)$, then

$$
W(k, h)=\frac{\delta(k-p)\left(\lambda^{h}\right)}{h !},
$$

where $p$ and $q$ are positive and $\lambda, a, b$ are scalars.

Theorem 2. If $u(x, t)=D_{x}^{q} v(x, t)$ and $\beta \in N$ is order of fractional, then

$$
U(k, h)=\frac{\Gamma(q+1+k / \beta)}{\Gamma(1+k / \beta)} V(k+\beta q, h) .
$$

Definition 3. The Beta function $B(a, b)$ of two variables is defined by

$$
B(a, b)=\int_{0}^{1}(1-x)^{a-1} x^{b-1} d x
$$

The following is proved easily:

$$
B(a, b)=\frac{\Gamma(a) \Gamma(b)}{\Gamma(a+b)} .
$$

Definition 4. The Kronecker delta function is given by

$$
\delta(k-m)= \begin{cases}1, & k=m, \\ 0, & k \neq m .\end{cases}
$$

\section{Main Theorem}

Now, we represent the main theorem of this study, through which a weakly singular Volterra integral equation can be expressed as a series of fractional differential transform for $w(x, t)=\int_{0}^{t} \int_{0}^{x}(x-\xi)^{p-1} N[\phi(\xi, \eta)] d \xi d \eta, 0<p<1, p \in Q$.

Theorem 5. Suppose that $\Phi(k, h)$ and $W(k, h)$ are the fractional differential transforms of the functions $\phi(x, t)$ and $w(x, t)$, respectively, such that

$$
\begin{aligned}
& w(x, t)=\int_{0}^{t} \int_{0}^{x}(x-\xi)^{p-1} N[\phi(\xi, \eta)] d \xi d \eta, \\
& 0<p<1, p \in Q .
\end{aligned}
$$

Then, by choosing a suitable $\beta \in z^{+}$such that $\beta p \in z^{+}$, we have

$$
\begin{aligned}
W(k, h)= & \frac{1}{((h-q) / \alpha)+1} \sum_{l=0}^{k} \delta(l-\beta p) \\
& \cdot \sum_{q=0}^{h} \delta(q-\alpha) B\left(\frac{k-l}{\beta}+1, p\right) \\
& \cdot \Phi(k-l, h-q),
\end{aligned}
$$

where $B(\cdot, \cdot)$ is the Beta function and $\delta$ is the Kronecker delta function.

Proof. By putting $N[\phi(x, t)]=\sum_{k=0}^{\infty} \sum_{h=0}^{\infty} \Phi(k, h) x^{k / \beta} t^{h / \alpha}$ in (22), it will change into

$$
\begin{aligned}
& w(x, t)=\int_{0}^{t} \int_{0}^{x}(x-\xi)^{p-1} N[\phi(\xi, \eta)] d \xi d \eta \\
& =\int_{0}^{t}\left(\int_{0}^{x}(x-\xi)^{p-1} \sum_{k=0}^{\infty} \sum_{h=0}^{\infty} \Phi(k, h) \xi^{k / \beta} \eta^{h / \alpha} d \xi\right) d \eta \\
& =\sum_{k=0}^{\infty} \sum_{h=0}^{\infty} \Phi(k, h) \int_{0}^{t}\left(\int_{0}^{x}(x-\xi)^{p-1} \xi^{k / \beta} d \xi\right) \eta^{h / \alpha} d \eta .
\end{aligned}
$$

To calculate $\int_{0}^{x}(x-\xi)^{p-1} \xi^{k / \beta} d \xi$, we change variable $x / \xi=v$ and according to Definition 3 we get

$$
\int_{0}^{x}(x-\xi)^{p-1} \xi^{k / \beta} d \xi=B\left(\frac{k}{\beta}+1, p\right) x^{p+k / \beta} .
$$

The following equation is obtained by using Theorem 1 and replacing (25) into (24):

$$
\begin{aligned}
w & (x, t) \\
& =\sum_{k=0}^{\infty} \sum_{h=0}^{\infty} \Phi(k, h) B\left(\frac{k}{\beta}+1, p\right) x^{p+k / \beta} \int_{0}^{t} \eta^{h / \alpha} d \eta .
\end{aligned}
$$

Hence

$$
\begin{gathered}
w(x, t)=\frac{1}{(h / \alpha)+1} x^{p} \sum_{k=0}^{\infty} \sum_{h=0}^{\infty} B\left(\frac{k}{\beta}+1, p\right) \Phi(k, h) \\
\cdot x^{k / \beta} t^{h / \alpha+1}=\frac{1}{(h / \alpha)+1} \sum_{l=0}^{\infty} \delta(l-\beta p)
\end{gathered}
$$




$$
\begin{aligned}
& \cdot x^{l / \beta} \sum_{q=0}^{\infty} \delta(q-\alpha) t^{q / \alpha} \\
& \cdot \sum_{k=0}^{\infty} \sum_{h=0}^{\infty} B\left(\frac{k}{\beta}+1, p\right) \Phi(k, h) x^{k / \beta} t^{h / \alpha} \\
& =\frac{1}{(h / \alpha)+1} \\
& \cdot \sum_{k=0}^{\infty} \sum_{h=0}^{\infty} \sum_{l=0}^{\infty} \delta(l-\beta p) \sum_{q=0}^{\infty} \delta(q-\alpha) B\left(\frac{k}{\beta}+1, p\right) \\
& \cdot \Phi(k, h) x^{(k+l) / \beta} t^{(h+q) / \alpha}=\frac{1}{((h-q) / \alpha)+1} \\
& \cdot \sum_{k=0}^{\infty} \sum_{h=0}^{\infty} \sum_{l=0}^{k} \delta(l-\beta p) \sum_{q=0}^{h} \delta(q-\alpha) B\left(\frac{k-l}{\beta}+1, p\right) \\
& . \Phi(k-l, h-q) x^{k / \beta} t^{h / \alpha} .
\end{aligned}
$$

According to $w(x, t)=\sum_{k=0}^{\infty} \sum_{h=0}^{\infty} W(k, h) x^{k / \beta} t^{h / \alpha}$, one can conclude that

$$
\begin{aligned}
& W(k, h)=\frac{1}{((h-q) / \alpha)+1} \sum_{l=0}^{k} \delta(l-\beta p) \\
& \cdot \sum_{q=0}^{h} \delta(q-\alpha) B\left(\frac{k-l}{\beta}+1, p\right) \Phi(k-l, h-q) .
\end{aligned}
$$

The proof is completed.

\section{Description of Method}

In this section, we try to describe the FDTM for (1) and initial condition (2). Based on Theorems 1, 2, and 5, FDTM for (1) and (2) would result as follows:

$$
\begin{aligned}
& (1+h) \Phi(k, h+1)=\Phi(k, h)+F(k, h) \\
& +\frac{1}{((h-q) / \alpha)+1} \sum_{l=0}^{k} \delta(l-\beta p) \\
& \quad \cdot \sum_{q=0}^{h} \delta(q-\alpha) B\left(\frac{k-l}{\beta}+1, p\right) \Phi(k-l, h-q) . \\
& \Phi(k, h)=\Phi(k, 0), \quad k=0,1,2,3, \ldots,
\end{aligned}
$$

in which $\Phi(k, h)$ and $F(k, h)$ are the FDTM of $\phi(x, t)$ and $f(x, t)$, respectively. Then according to the recurrence relation (11) the unknown function would result.

\section{Applications}

In this section, we take some examples to clarify the advantages and the accuracy of the fractional differential transform method (FDTM) for solving a kind of nonlinear partial integro-differential equation with a weakly singular kernel.
For each of these examples, we obtain a recurrence relation. In all of the examples, we choose $\alpha=1$ and $\beta$ is chosen in a way where $\beta p \in z^{+}$.

Example 1. Consider the following nonlinear partial integrodifferential equation with a weakly singular kernel with $p=$ $1 / 2[23]$ :

$$
\begin{aligned}
\phi_{t}= & \phi(x, t)+f(x, t) \\
& +\int_{0}^{t} \int_{0}^{x}(x-\xi)^{p-1}[\phi(\xi, \eta)]^{2} d \xi d \eta,
\end{aligned}
$$

with the initial condition $\phi(x, 0)=x^{2}$ and $f(x, t)=2 t-x^{2}-$ $t^{2}-(2 / 315) x^{1 / 2} t\left(128 x^{4}+112 x^{2} t^{2}+63 t^{4}\right)$

For solving (30), we employ the FDTM, to get

$$
\begin{aligned}
& (1+h) \Phi(k, h+1)=\Phi(k, h)+2 \delta(h-1) \delta(k) \\
& -\delta(h) \delta(k-4)-\delta(h-2) \delta(k)-\frac{256}{315} \delta(h-1) \\
& \cdot \delta(k-9)-\frac{224}{315} \delta(h-3) \delta(k-5)-\frac{126}{315} \delta(h-5) \\
& \cdot \delta(k-1)+\frac{1}{h} B\left(\frac{k-l}{2}+1, \frac{1}{2}\right) \\
& \quad \cdot \sum_{s=0}^{k-1} \sum_{r=0}^{h-1} \Phi(s, h-1-r) \Phi(k-1-s, r),
\end{aligned}
$$

where $h, k \geq 1$ in the upper bound of the sigmas and the differential transform of initial condition is as follows:

$$
\begin{aligned}
& \Phi(k, 0)=\delta(k-4)=\left\{\begin{array}{ll}
1 & k=4 \\
0 & k \neq 4
\end{array} \longrightarrow\right. \\
& \Phi(4,0)=1 .
\end{aligned}
$$

Also we have

$$
\begin{aligned}
\phi(x, t)= & \sum_{k=0}^{\infty} \sum_{h=0}^{\infty} \Phi(k, h) x^{k / 2} t^{h} \\
= & \Phi(0,0)+\Phi(0,1) t+\Phi(0,2) t^{2}+\cdots \\
& +\Phi(1,0) x^{1 / 2}+\Phi(1,1) x^{1 / 2} t \\
& +\Phi(1,2) x^{1 / 2} t^{2}+\Phi(1,3) x^{1 / 2} t^{3}+\cdots \\
& +\Phi(2,0) x+\Phi(2,1) x t+\Phi(2,2) x t^{2} \\
& +\Phi(2,3) x t^{3}+\cdots+\Phi(3,0) x^{3 / 2} \\
& +\Phi(3,1) x^{3 / 2} t+\Phi(3,2) x^{3 / 2} t^{2} \\
& +\Phi(3,3) x^{3 / 2} t^{3}+\cdots+\Phi(4,0) x^{2} \\
& +\Phi(4,1) x^{2} t+\Phi(4,2) x^{2} t^{2}+\Phi(4,3) x t^{3} \\
& +\cdots
\end{aligned}
$$


By using the recurrence relation (31) and the transform initial condition (32), we get the following:

$$
\begin{aligned}
& k=0 \\
& h=0
\end{aligned}
$$

$\downarrow$

$$
\begin{gathered}
\Phi(0,1)=\Phi(0,0)+0 \\
\downarrow \\
\Phi(0,1)=0 ; \\
k=1 \\
h=0 \\
\downarrow
\end{gathered}
$$$$
\Phi(1,1)=\Phi(1,0)+0
$$$$
\downarrow
$$

$$
\begin{array}{r}
\Phi(1,1)=0 ; \\
k=2 \\
h=0
\end{array}
$$$$
\downarrow
$$$$
\Phi(2,1)=\Phi(2,0)+0
$$

\section{$\downarrow$}

$\Phi(2,1)=0$

$$
k=3
$$$$
h=0
$$

$\Phi(3,1)=\Phi(3,0)+0$

$\downarrow$

$$
\begin{aligned}
\Phi(3,1) & =0 ; \\
k & =4 \\
h & =0
\end{aligned}
$$$$
\downarrow
$$

$$
\begin{gathered}
\Phi(4,1)=\Phi(4,0)-1 \\
\downarrow \\
\Phi(4,1)=0,
\end{gathered}
$$

and by applying the same calculations, the following can be concluded:

$$
\Phi(k, 1)=0 \quad k \geq 5 \text {. }
$$

Also we put

$$
\begin{aligned}
& k=0 \\
& h=1 \\
& \downarrow \\
& 2 \Phi(0,2)=\Phi(0,1)+2 \\
& \downarrow \\
& \Phi(0,2)=1 ; \\
& k=1 \\
& h=1 \\
& \downarrow \\
& 2 \Phi(1,2)=\Phi(1,1)+0 \\
& \downarrow \\
& \Phi(1,2)=0 \text {; } \\
& k=2 \\
& h=1 \\
& \downarrow \\
& 2 \Phi(2,2)=\Phi(2,1)+0 \\
& \downarrow \\
& \Phi(2,2)=0 \\
& \vdots \\
& k=9 \\
& h=1 \\
& \downarrow \\
& 2 \Phi(9,2)=\Phi(9,1)-\frac{2 \times 128}{315} \\
& +B\left(4+1, \frac{1}{2}\right) \sum_{s=0}^{9-1} \sum_{r=0}^{0} \Phi(s, 0) \Phi(8-s, 0) .
\end{aligned}
$$

By Definition 3, we have

$$
B\left(5, \frac{1}{2}\right)=\frac{2 \times 128}{315} .
$$

So $\Phi(9,2)=0$.

And by applying the same calculations, we can conclude that

$$
\Phi(k, 2)=0 \quad k>9 .
$$

By continuing this process, we can also conclude the following:

$$
\Phi(k, h)=0 \quad k \geq 0, h \geq 3 .
$$


Therefore, by substituting the above values into (33), the exact solution is obtained in the following form:

$$
\phi(x, t)=x^{2}+t^{2}
$$

which is the particular solution obtained in [23].

Example 2. Consider the following nonlinear partial integrodifferential equation with a weakly singular kernel:

$$
\begin{aligned}
\phi_{t}= & \phi(x, t)+f(x, t) \\
& +\int_{0}^{t} \int_{0}^{x}(x-\xi)^{-1 / 4}[\phi(\xi, \eta)]^{2} d \xi d \eta
\end{aligned}
$$

with the initial condition $\phi(x, 0)=x$ and $f(x, t)=-x \sin t-$ $x \cos t-(64 / 231) x^{11 / 4} t-(32 / 231) x^{11 / 4} \sin 2 t$.

Taking into consideration the two-dimensional transform for (41) and the related theorems, we have

$$
\begin{aligned}
& (h+1) \Phi(k, h+1)=\Phi(k, h)-\delta(k-4) \frac{1}{h !} \sin \frac{h \pi}{2} \\
& -\delta(k-4) \frac{1}{h !} \cos \frac{h \pi}{2}-\frac{64}{231} \delta(k-11) \delta(h-1) \\
& -\frac{32}{231}\left(\frac{2^{h}}{h !}\right) \sin \frac{h \pi}{2} \delta(k-11)+\frac{1}{h} \\
& \cdot B\left(\frac{k-3}{4}+1, \frac{3}{4}\right) \\
& \quad \sum_{s=0}^{k-3} \sum_{r=0}^{h-1} \Phi(s, h-1-r) \Phi(k-3-s, r) .
\end{aligned}
$$

The differential transform of the initial condition is as follows:

$$
\Phi(k, 0)=\delta(k-4)=\left\{\begin{array}{ll}
1 & k=4 \\
0 & k \neq 4
\end{array} \longrightarrow \Phi(4,0)=1 .\right.
$$

Also we have

$$
\begin{aligned}
\phi(x, t)= & \sum_{k=0}^{\infty} \sum_{h=0}^{\infty} \Phi(k, h) x^{k / 4} t^{h} \\
= & \Phi(0,0)+\Phi(0,1) t+\Phi(0,2) t^{2}+\cdots \\
& +\Phi(1,0) x^{1 / 4}+\Phi(1,1) x^{1 / 4} t \\
& +\Phi(1,2) x^{1 / 4} t^{2}+\Phi(1,3) x^{1 / 4} t^{3}+\cdots \\
& +\Phi(2,0) x^{2 / 4}+\Phi(2,1) x^{2 / 4} t \\
& +\Phi(2,2) x^{2 / 4} t^{2}+\Phi(2,3) x^{2 / 4} t^{3}+\cdots \\
& +\Phi(3,0) x^{3 / 4}+\Phi(3,1) x^{3 / 4} t \\
& +\Phi(3,2) x^{3 / 4} t^{2}+\Phi(3,3) x^{3 / 4} t^{3}+\cdots \\
& +\Phi(4,0) x+\Phi(4,1) x t+\Phi(4,2) x t^{2} \\
& +\Phi(4,3) x t^{3}+\cdots
\end{aligned}
$$

By using the recurrence relation (42) and the differential transform of initial condition (43), we get

$$
\begin{aligned}
& k=0 \\
& h=0 \\
& \downarrow \\
& \Phi(0,1)=\Phi(0,0)-0 \\
& \downarrow \\
& \Phi(0,1)=0 \text {; } \\
& k=1 \\
& h=0 \\
& \downarrow \\
& \Phi(1,1)=\Phi(1,0)-0 \\
& \downarrow \\
& \Phi(1,1)=0 \text {; } \\
& k=2 \\
& h=0 \\
& \downarrow \\
& \Phi(2,1)=\Phi(2,0)-0 \\
& \downarrow \\
& \Phi(2,1)=0 \text {; } \\
& k=3 \\
& h=0 \\
& \Phi(3,1)=\Phi(3,0)-0 \\
& \downarrow \\
& \Phi(3,1)=0 \text {; } \\
& k=4 \\
& h=0 \\
& \downarrow \\
& \Phi(4,1)=\Phi(4,0)-1 \\
& \downarrow \\
& \Phi(4,1)=0
\end{aligned}
$$

and by applying the same calculations, it can be concluded that

$$
\begin{aligned}
& \Phi(k, 1)=0 \quad k \geq 5 ; \\
& k=0
\end{aligned}
$$




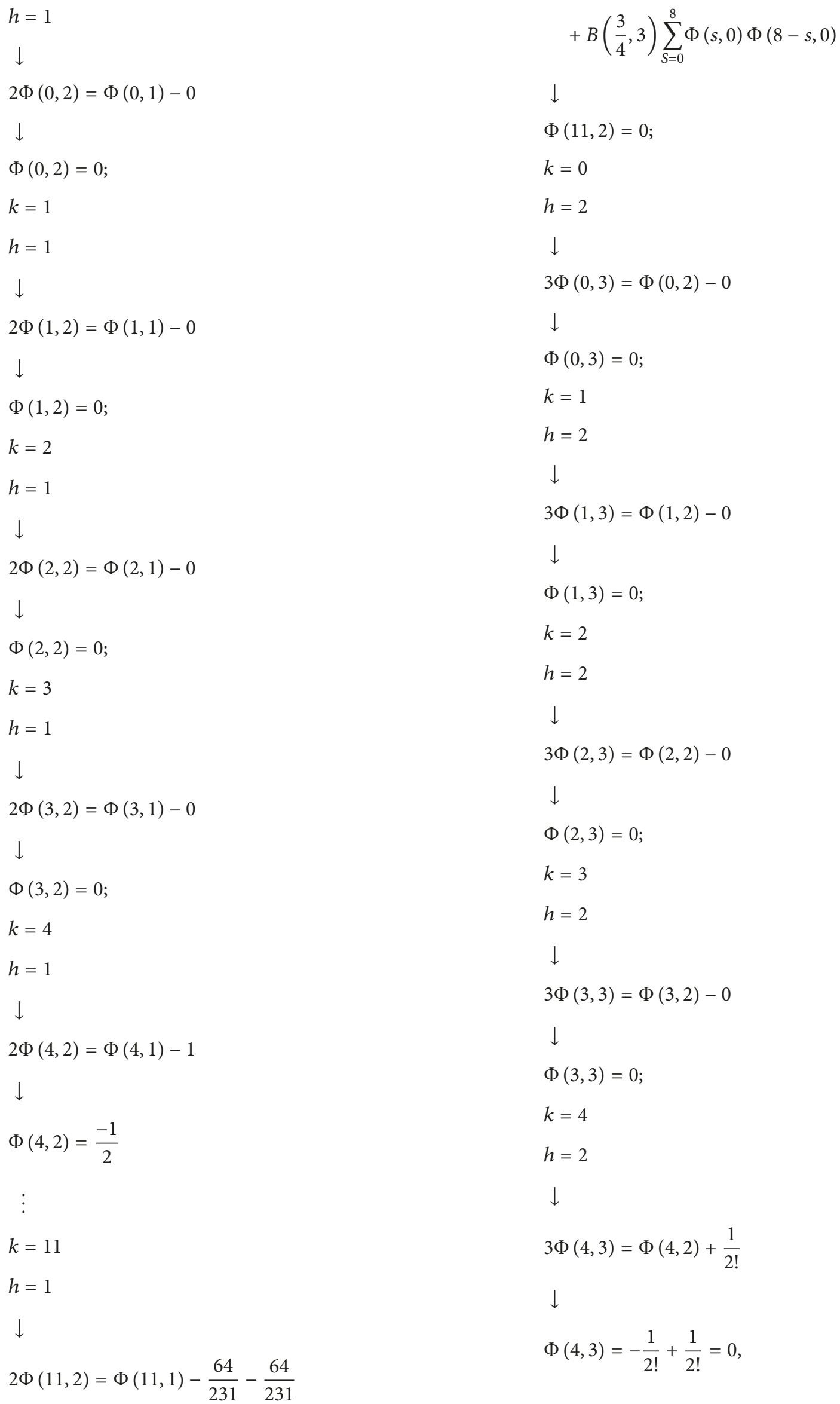


and by applying the same calculations, we conclude the following:

$$
\begin{gathered}
\Phi(k, 3)=0 \quad k \geq 5 ; \\
k=0 \\
h=3 \\
\downarrow
\end{gathered}
$$$$
4 \Phi(0,4)=\Phi(0,3)-0
$$$$
\downarrow
$$

$$
\begin{aligned}
\Phi(0,4) & =0 ; \\
k & =1 \\
h & =3
\end{aligned}
$$

$$
\begin{aligned}
& \downarrow \\
4 \Phi(1,4) & =\Phi(1,3)-0 \\
\downarrow & \downarrow \\
\Phi(1,4) & =0 ; \\
k & =2 \\
h & =3 \\
& \downarrow \\
4 \Phi(2,4) & =\Phi(2,3)-0
\end{aligned}
$$

$\downarrow$

$$
\begin{aligned}
\Phi(2,4) & =0 ; \\
k & =3 \\
h & =3 \\
& \downarrow \\
4 \Phi(3,4) & =\Phi \\
\downarrow & \\
\Phi(3,4) & =0 ; \\
k & =4 \\
h & =3
\end{aligned}
$$$$
4 \Phi(3,4)=\Phi(3,3)-0
$$

\section{$\downarrow$}

$$
4 \Phi(4,4)=\Phi(4,3)+\frac{1}{3 !}
$$

$\Phi(4,4)=\frac{1}{4 !}$

$\Phi(k, 4)=0 \quad k \geq 5$.
Therefore, by substituting the above values into (44), the exact solution is obtained in the following form:

$$
\begin{aligned}
\phi(x, t)= & \Phi(4,0) x+\Phi(4,1) x t+\Phi(4,2) x t^{2} \\
& +\Phi(4,3) x t^{3}+\cdots \\
= & x-\frac{1}{2 !} x t^{2}+\frac{1}{4 !} x t^{4}+\cdots=x \cos t .
\end{aligned}
$$

Example 3. Consider the following nonlinear partial integrodifferential equation with a weakly singular kernel:

$$
\begin{aligned}
\phi_{t}= & \phi(x, t)+f(x, t) \\
& +\int_{0}^{t} \int_{0}^{x}(x-\xi)^{-3 / 5}[\phi(\xi, \eta)]^{2} d \xi d \eta
\end{aligned}
$$

with the initial condition $\phi(x, 0)=x$ and $f(x, t)=$ $(125 / 168) x^{12 / 5} e^{2 t}$.

To solve (49), by applying FDTM, we have

$$
\begin{aligned}
& (h+1) \Phi(k, h+1)=\Phi(k, h)-\frac{125}{168} \delta(k-12) \frac{2^{h}}{h !} \\
& +\frac{1}{h} B\left(\frac{k-2}{5}+1, \frac{2}{5}\right) \\
& \quad \cdot \sum_{s=0}^{k-2} \sum_{r=0}^{h-1} \Phi(s, h-1-r) \Phi(k-2-s, r),
\end{aligned}
$$

where $k \geq 2, h \geq 1$ in the upper bound of the sigmas and differential transform of initial condition is as follows:

$$
\Phi(k, 0)=\delta(k-5)=\left\{\begin{array}{ll}
1 & k=5 \\
0 & k \neq 5
\end{array} \longrightarrow \Phi(5,0)=1\right.
$$

Also we have

$$
\begin{aligned}
\phi(x, t)= & \sum_{k=0}^{\infty} \sum_{h=0}^{\infty} \Phi(k, h) x^{k / 5} t^{h} \\
= & \Phi(0,0)+\Phi(0,1) t+\Phi(0,2) t^{2}+\cdots \\
& +\Phi(1,0) x^{1 / 5}+\Phi(1,1) x^{1 / 5} t \\
& +\Phi(1,2) x^{1 / 5} t^{2}+\Phi(1,3) x^{1 / 5} t^{3}+\cdots \\
& +\Phi(2,0) x^{2 / 5}+\Phi(2,1) x^{2 / 5} t \\
& +\Phi(2,2) x^{2 / 5} t^{2}+\Phi(2,3) x^{2 / 5} t^{3}+\cdots \\
& +\Phi(3,0) x^{3 / 5}+\Phi(3,1) x^{3 / 5} t \\
& +\Phi(3,2) x^{3 / 5} t^{2}+\Phi(3,3) x^{3 / 5} t^{3}+\cdots \\
& +\Phi(4,0) x^{4 / 5}+\Phi(4,1) x^{4 / 5} t \\
& +\Phi(4,2) x^{4 / 5} t^{2}+\Phi(4,3) x^{4 / 5} t^{3}+\cdots \\
& +\Phi(5,0) x+\Phi(5,1) x t+\Phi(5,2) x t^{2} \\
& +\Phi(5,3) x t^{3}+\cdots
\end{aligned}
$$


By using the recurrence relation (50), the differential transform of initial condition (51), and the same calculations of the above-mentioned examples, it is concluded that

$$
\begin{aligned}
\phi(x, t)= & x+x t+\frac{1}{2 !} x t^{2}+\frac{1}{3 !} x t^{3}+\cdots \\
& -\frac{125}{168} x^{12 / 5}\left(t+\frac{t^{2}}{2 !}+\frac{t^{3}}{3 !}+\cdots\right) .
\end{aligned}
$$

Of course this solution is an analytical solution.

\section{Conclusion}

In this paper, we have described the definition and operation of two-dimensional fractional differential transform; fractional derivatives have been considered in the Caputo and Riemann-Liouville sense and the main theorem on fractional differential transform method. Using the fractional differential transform method, a kind of nonlinear partial integro-differential equation with a singular kernel was solved approximately and analytically. We have used FDTM in this paper to solve (30) which was solved by operational matrices in [23]. The advantages of this method are that one obtains satisfactory results in less time, there is no need to calculate any repeated integral, and there is no discretization.

\section{Conflicts of Interest}

The authors declare that there are no conflicts of interest regarding the publication of this paper.

\section{References}

[1] G. Capobianco, D. Conte, and I. Del Prete, "High performance parallel numerical methods for Volterra equations with weakly singular kernels," Journal of Computational and Applied Mathematics, vol. 228, no. 2, pp. 571-579, 2009.

[2] J. A. Dixon, "A nonlinear weakly singular Volterra integrodifferential equation arising from a reaction-diffusion study in a small cell," Journal of Computational and Applied Mathematics, vol. 18, no. 3, pp. 289-305, 1987.

[3] P. Linz, Analytical and Numerical Methods for Volterra Equations, vol. 7 of SIAM Studies in Applied Mathematics, Society for Industrial and Applied Mathematics (SIAM), Philadelphia, Pa, USA, 1985.

[4] J. B. Keller and W. E. Olmstead, "Temperature of a nonlinearly radiating semi-infinite solid," Quarterly of Applied Mathematics, vol. 29, pp. 559-566, 1971/72.

[5] A. Palamara Orsi, "Product integration for Volterra integral equations of the second kind with weakly singular kernels," Mathematics of Computation, vol. 65, no. 215, pp. 1201-1212, 1996.

[6] Y. Chen and T. Tang, "Spectral methods for weakly singular Volterra integral equations with smooth solutions," Journal of Computational and Applied Mathematics, vol. 242, pp. 53-69, 2013.

[7] H. Brunner, A. Pedas, and G. Vainikko, "The piecewise polynomial collocation method for nonlinear weakly singular Volterra equations," Mathematics of Computation, vol. 68, no. 227, pp. 1079-1095, 1999.
[8] J. C. Lopez Marcos, "A difference scheme for a nonlinear partial integrodifferential equation," SIAM Journal on Numerical Analysis, vol. 27, no. 1, pp. 20-31, 1990.

[9] L. Bougoffa, R. C. Rach, and A. Mennouni, "An approximate method for solving a class of weakly-singular Volterra integrodifferential equations," Applied Mathematics and Computation, vol. 217, no. 22, pp. 8907-8913, 2011.

[10] M. Dehghan, "Solution of a partial integro-differential equation arising from viscoelasticity," International Journal of Computer Mathematics, vol. 83, no. 1, pp. 123-129, 2006.

[11] P. Baratella and A. P. Orsi, "A new approach to the numerical solution of weakly singular Volterra integral equations," Journal of Computational and Applied Mathematics, vol. 163, no. 2, pp. 401-418, 2004.

[12] T. Diogo and P. Lima, "Superconvergence of collocation methods for a class of weakly singular Volterra integral equations," Journal of Computational and Applied Mathematics, vol. 218, no. 2, pp. 307-316, 2008.

[13] T. Tang, "A finite difference scheme for partial integrodifferential equations with a weakly singular kernel," Applied Numerical Mathematics, vol. 11, no. 4, pp. 309-319, 1993.

[14] Y. Chen and T. Tang, "Spectral methods for weakly singular Volterra integral equations with smooth solutions," Journal of Computational and Applied Mathematics, vol. 233, no. 4, pp. 938-950, 2009.

[15] A. Arikoglu and I. Ozkol, "Solution of fractional differential equations by using differential transform method," Chaos, Solitons \& Fractals, vol. 34, no. 5, pp. 1473-1481, 2007.

[16] A. Arikoglu and I. Ozkol, "Solution of fractional integrodifferential equations by using fractional differential transform method," Chaos, Solitons \& Fractals, vol. 40, no. 2, pp. 521-529, 2009.

[17] S. Momani and Z. Odibat, "A novel method for nonlinear fractional partial differential equations: combination of DTM and generalized Taylor's formula," Journal of Computational and Applied Mathematics, vol. 220, no. 1-2, pp. 85-95, 2008.

[18] J. K. Zhou, differential transformation and its applications for electrical circuits, Huazhong University Press, Wuha, China, 1986.

[19] R. Hilfer, Applications of Fractional Calculus in Physics, World Scientific, Singapore, 2000.

[20] E. Tohidi and F. Toutounian, "Convergence analysis of Bernoulli matrix approach for one-dimensional matrix hyperbolic equations of the first order," Computers \& Mathematics with Applications, vol. 68, no. 1-2, pp. 1-12, 2014.

[21] F. Toutounian and E. Tohidi, "A new Bernoulli matrix method for solving second order linear partial differential equations with the convergence analysis," Applied Mathematics and Computation, vol. 223, pp. 298-310, 2013.

[22] G. Fairweather, "Spline collocation methods for a class of hyperbolic partial integro-differential equations," SIAM Journal on Numerical Analysis, vol. 31, no. 2, pp. 444-460, 1994.

[23] S. Singh, V. K. Patel, V. K. Singh, and E. Tohidi, "Numerical solution of nonlinear weakly singular partial integro-differential equation via operational matrices," Applied Mathematics and Computation, vol. 298, pp. 310-321, 2017.

[24] S. Singh, V. K. Patel, and V. K. Singh, "Operational matrix approach for the solution of partial integro-differential equation," Applied Mathematics and Computation, vol. 283, pp. 195207, 2016. 
[25] W. McLean, I. H. Sloan, and V. Thomee, “Time discretization via Laplace transformation of an integro-differential equation of parabolic type," Numerische Mathematik, vol. 102, no. 3, pp. 497-522, 2006.

[26] E. Rahimi, H. Taghvafard, and G. H. Erjaee, "Fractional differential transform method for solving a class of weakly singular Volterra integral equations," Iranian Journal of Science \& Technology, vol. 38, no. 1, pp. 69-73, 2014.

[27] D. Nazari and S. Shahmorad, "Application of the fractional differential transform method to fractional-order integrodifferential equations with nonlocal boundary conditions," Journal of Computational and Applied Mathematics, vol. 234, no. 3, pp. 883-891, 2010.

[28] A. A. Kilbas, H. M. Srivastava, and J. J. Trujillo, Theory and Applications of Fractional Differential Equations, New York, NY, USA, Elsevier, 2006.

[29] M. Sen, Introduction to fractional-order operators and their engineering applications, Department of Aerospace and Mechanical Engineering University of Notre Dame, 2014.

[30] Z. M. Odibat and N. T. Shawagfeh, "Generalized Taylor's formula," Applied Mathematics and Computation, vol. 186, no. 1, pp. 286-293, 2007.

[31] M. Caputo, "Linear models of dissipation whose $Q$ is almost frequency independent-II," The Geophysical Journal of the Royal Astronomical Society, vol. 13, no. 5, pp. 529-539, 1967. 


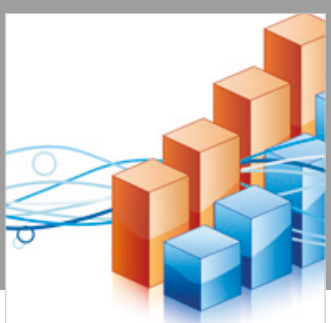

Advances in

Operations Research

\section{-n-m}
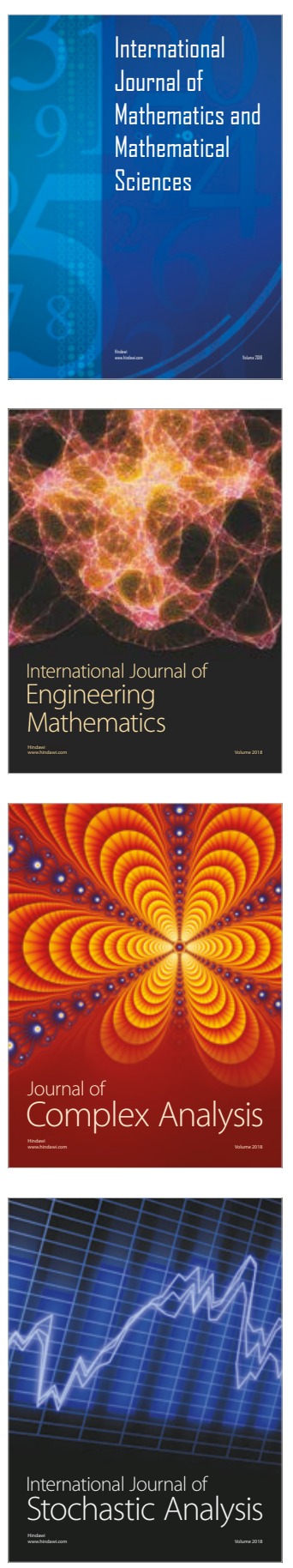
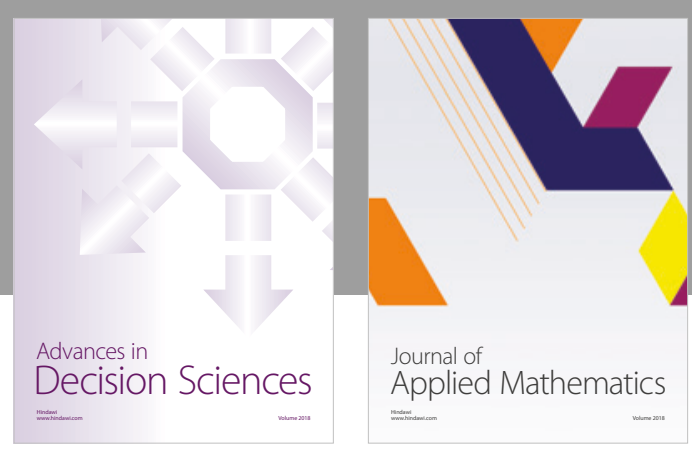

Journal of

Applied Mathematics
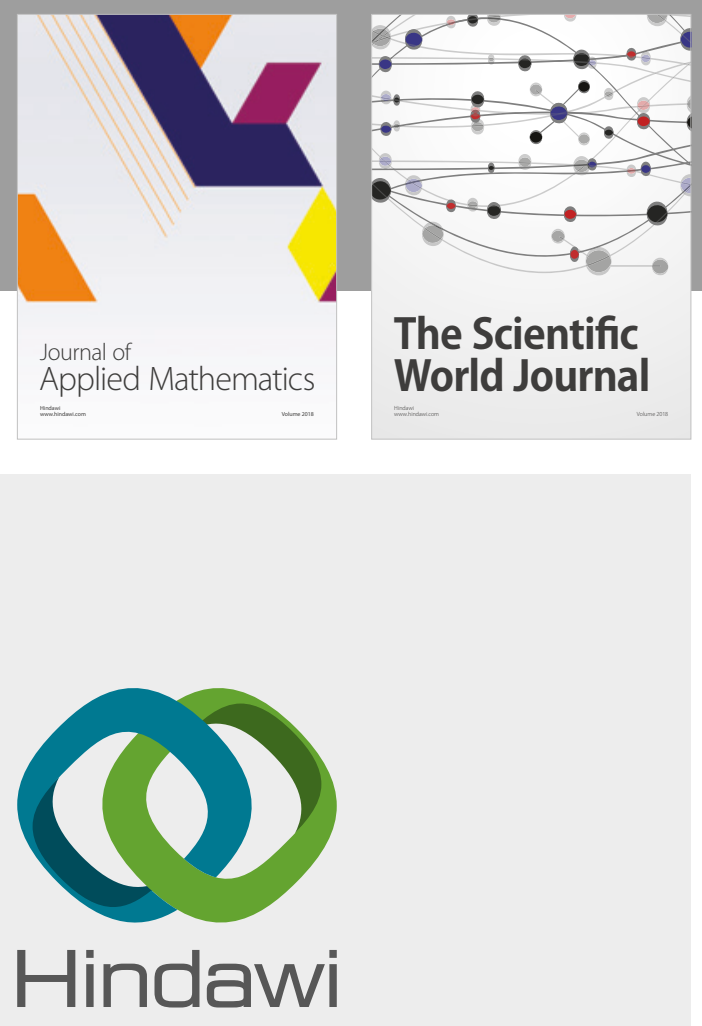

Submit your manuscripts at

www.hindawi.com

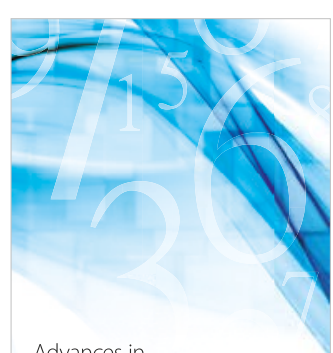

Advances in
Numerical Analysis
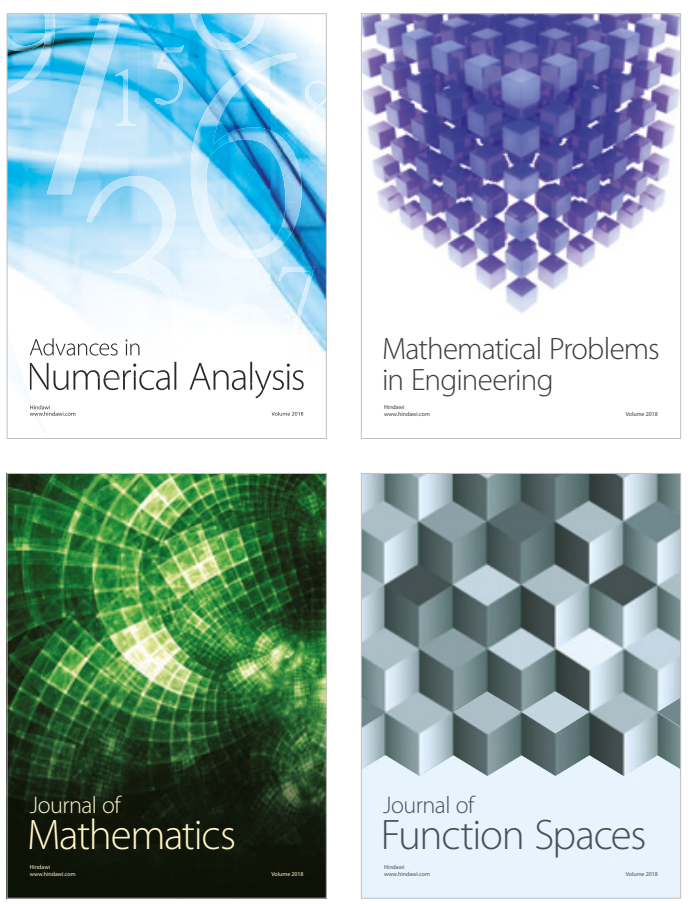

Mathematical Problems in Engineering

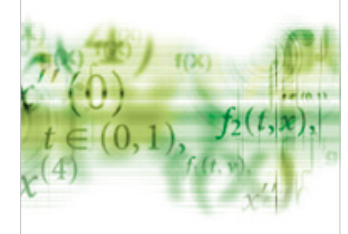

International Journal of

Differential Equations

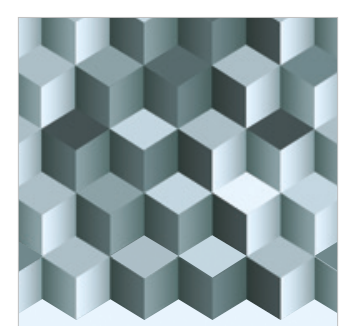

Journal of

Function Spaces

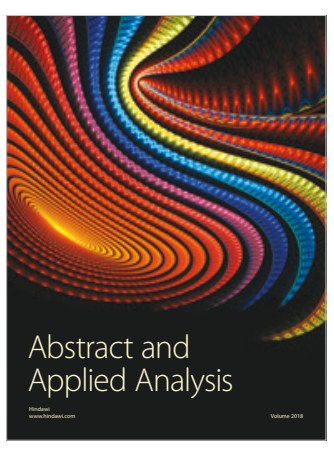

The Scientific

World Journal

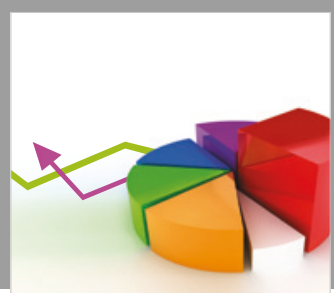

Journal of

Probability and Statistics
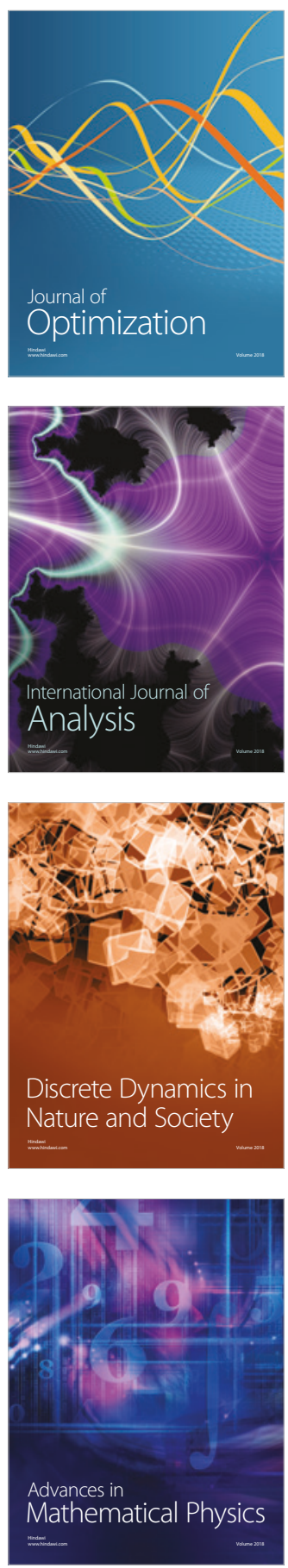